\title{
Degenerative lumbar intervertebral instability: what is it and how does imaging contribute?
}

\author{
Antonio Leone • Victor N. Cassar-Pullicino • \\ Giuseppe Guglielmi • Lorenzo Bonomo
}

Published online: 6 February 2009

(C) ISS 2009

\section{Introduction}

Although low back pain (LBP) is the most common rheumatologic symptom that leads to consultation with a general practitioner, the identification of a specific symptomatic cause remains elusive in the majority of patients with mechanical LBP. Degenerative lumbar intervertebral instability as a cause of chronic LBP is an important factor in determining indications for spinal surgery.

The concept of lumbar instability remains a subject of considerable debate. In spite of many efforts, a widely accepted definition of the condition has not been clearly established. The best diagnostic methods and the most efficacious treatment approaches are controversial, and the relationship between radiologic evidence of instability and symptom production is not always clear cut.

A. Leone $(\bowtie) \cdot$ L. Bonomo

Department of Bioimaging and Radiological Sciences,

School of Medicine, Catholic University,

Largo A. Gemelli 1,

00168 Rome, Italy

e-mail: a.leonemd@tiscali.it

V. N. Cassar-Pullicino

Department of Diagnostic Imaging,

The Robert Jones and Agnes Hunt Orthopaedic and District

Hospital,

Oswestry,

Shopshire, UK

G. Guglielmi

Department of Radiology,

Scientific Institute Casa Sollievo della Sofferenza,

San Giovanni Rotondo and University of Foggia,

Foggia, Italy
This perspective discusses the role of imaging as a potential unifier of varied clinical labels and therapeutic options.

\section{Pathomechanisms}

A workable definition of instability is based on a loss of motion segment stiffness, such that force application to that segment produces greater displacement than would be seen in a normal state $[1,2]$. In a biomechanical sense, stiffness is defined as the ratio of the load applied to a structure and the resulting motion. Stability to the lumbar spine is provided by the restraining structures working in conjunction (i.e., disks, joints, ligaments, and muscles) [3]. Degenerative processes, leading to laxity of restraining structures, result in altered equilibrium and instability [26]. The degenerative processes of the lumbar spine generally initiate from the intervertebral disk with progressive biochemical and structural changes leading to a modification in its physical properties of elasticity and mechanical resistance and eventual collapse of the intervertebral disk.

Three clinically relevant consequences of acquired collapse of the intervertebral disk are: (a) pathologic changes in the vertebral bodies, with osteophyte development; (b) anterior bulging of the flaval ligaments and posterior bulging of the posterior longitudinal ligament, with consequential narrowing of the central spinal canal; and (c) posterior bulging of redundant posterior disk surface, with narrowing of the central spinal canal and of the inferior recesses of the neural foramina. Moreover, weakening of the disk structure permits the adjacent vertebrae to slide back and forth over each other, resulting in laxity of the ligamentous network binding the vertebrae 
together and leading to cranio-caudal partial subluxation of the facet joints which may be asymmetric from side to side. Subsequent stresses on the facet joints will give rise to osteoarthritis with ostheophytosis, cartilage degeneration, and synovial inflammation. Osteoarthritis of the facet joints, which may occur independently of the disk, may allow hypermobility of the facet joint and then may lead to a degenerative spondylolisthesis [1]. Grobler et al. [7] demonstrated a significant increase in sagittal orientation of L4-5 facet joints in patients with degenerative spondylolisthesis compared with a normal population. This sagittal orientation facilitates vertebral slippage when the other predisposing factors are present. Because of these abnormalities and the preponderance of coronal orientation of the L5-S1 facet joints, the majority of degenerative spondylolisthesis occurs at the L4-5 level [8].

The relationship between lumbar instability and degenerative spondylolisthesis was suggested by Kirkaldy-Willis and Farfan [4] who postulated three stages of lumbar spine degenerative changes with differing conditions of stability and motion: temporary dysfunction, unstable phase, and stabilization. The duration of each stage varies greatly, and there is no clear-cut division between the clinical signs and symptoms of one stage and the next. The first (temporary dysfunction) phase is associated with early degenerative changes and is assumed to transform into the second (unstable) phase in which the disk height is diminished, the ligaments and joint capsule are lax, and the facet joint cartilage degenerates. This then leads to a condition of segmental spinal instability, creating the basis for the deformity that is seen as the process transforms into the third (stabilization) phase. In this last stage, osteophytes and marked disk space narrowing lead to stabilization of the motion segment with a reduction (partial or complete) in its range of motion, sometimes after spondylolisthesis has already occurred. On the basis of this model, the radiologic observation of degenerative spondylolisthesis does not necessarily indicate that intervertebral instability is still present at the time of imaging because a re-stabilization may have already occurred.

Although several biomechanical and clinical studies [2, 6] have reported the association of disk degeneration with segmental instability, confirming Kirkaldy-Willis and Farfan's concept [4], this association was not confirmed in other studies $[9,10]$.

\section{Imaging evaluation}

The diagnosis of vertebral instability is commonly based on the imaging finding of abnormal vertebral motion. There may be abnormal translation and/or rotation around the $x$-, $y$-, and $z$-axes of the three-dimensional coordinates system proposed by Panjabi and White [11]. Vertebral instability is generally multidirectional, whereas the resulting displacement is evaluated in one plane at a time. Sagittal (front to back, or $z$-axis) and coronal (side to side, or $x$-axis) displacements are evaluated on radiographs, and displacements on the axial plane are evaluated on computed tomographic (CT) or magnetic resonance (MR) images. Differentiation between normal and abnormal motion, however, remains uncertain and challenging.

\section{Neutral radiographs}

Several radiographic findings have been proposed as indicators of vertebral instability. Moderate disk degeneration with mild disk space narrowing and osteosclerosis of vertebral end plates have been associated with vertebral instability. In contrast, a marked disk space narrowing has been considered to be indicative of the late stabilization phase [4]. The "traction" spur, which is located 2 or $3 \mathrm{~mm}$ from the endplate and has a horizontal orientation, and the intervertebral vacuum phenomenon also have been associated with vertebral instability [2]. Unfortunately, disk degeneration with mild disk space narrowing and osteosclerosis, intervertebral vacuum phenomenon, and "traction" spur are very common radiographic findings, and it seems unwarranted to recommend functional flexion-extension radiography for all patients demonstrating these findings. Moreover, the diagnostic value of these indirect signs of spinal instability remains unknown because their sensitivity and specificity cannot be determined in the absence of a well-defined reference standard [4].

\section{Functional radiography}

Functional radiography in the sagittal plane obtained in both flexion and extension allows measurement of the sagittal translation of a vertebra with respect to the underlying one and the amount of vertebral rotation in the sagittal plane (defined by the variation of the angle between two opposite vertebral endplates observed between the extremes of movement).

Functional flexion-extension radiography is the most widely used method in the imaging diagnosis of lumbar intervertebral instability [4, 5, 12, 13]. However, some investigators [14] have suggested that the use of functional flexion-extension radiography as the primary determinant of lumbar intervertebral instability should be questioned because of the lack of a non-traumatic and routinely applicable reference standard, inaccurate reproducibility, and non-standardized techniques.

The choice of patient position, lateral decubitus versus standing, which best optimizes the flexion-extension radiographs, has been subjective $[2,4,11,15]$; however, Wood 
et al. [15] recommended that flexion-extension radiographs should be performed in the lateral decubitus position. In their study, more abnormal translation was observed with the patient in this position than while standing. One possible explanation for their results could be that splinting of the spine from the paraspinal postural or abdominal musculature reduces the spine's range of motion when the patient is standing [4, 5]. In symptomatic patients, moreover, pain may act as check rein against bending of the trunk, resulting in underestimation of the true intervertebral motion [16].

The measurement technique described by Morgan and King [17] demonstrated the overall best performance and the least interference due to concomitant motions [18]. Other generally used techniques have been described by Posner et al. [19] and Dupuis et al. [5]; these techniques supposedly avoid inaccuracies that result from magnification by measuring translation as a percentage of vertebral body width. The cutoff between normal and abnormal movement is also difficult to determine. A large range of normal motion has been reported with a substantial overlap of symptomatic and asymptomatic motion patterns [16, 20]; sagittal rotation may be as high as $25^{\circ}$ in healthy young volunteers [20]. This hypermobility may or may not be pathologic, depending on the ability of the vertebral and soft tissue structures to accommodate the movement. However, values of $10^{\circ}$ for sagittal rotation and $4 \mathrm{~mm}$ for sagittal translation are typically used to infer instability [5, 17, 19]. Pathologic axial rotation can be detected on sidebending radiographs if the spinous processes move to the convex side, producing an asynchronous spinous process line $[4,13]$. Pathologic rotation can also manifest as a lateral translation (laterolisthesis) of one vertebra on another during lateral bending [1]. However, Pitkanen and Manninen [13] suggested that side-bending radiographs should not be routinely combined with flexion-extension radiographs in the radiologic diagnosis of lumbar instability. Side-bending radiographs complement flexion-extension radiographs and should be obtained if side-bending instability is clinically suspected, especially when flexion-extension radiographs are normal, but are unlikely to be helpful on a routine basis.

It can be concluded that the value of functional radiographs remains debatable; however, the majority of surgeons use them to disclose abnormal vertebral motion before deciding on surgical fusion.

\section{CT imaging}

CT can demonstrate underlying predisposing anatomic factors, such as facet joint asymmetry, which may lead to an abnormal axial rotation of a vertebra on the subjacent one (rotatory spondylolisthesis) [21]. The "twist test" is a technique of functional $\mathrm{CT}$ which may demonstrate increased abnormal motion, such as a gap of the facet joint space or an abnormal motion during rotation of the trunk, not clearly evident at functional radiography [4]. In the "twist test", the CT scan is obtained through the facet joint while the patient twists the torso and the pelvis is tightly strapped to the CT table. However, it is not known whether this technique allows the differentiation between normal and unstable spine [14].

\section{MR imaging}

MR imaging is established as the most reliable imaging method for diagnosing degenerative abnormalities of the lumbar spine and may influence indications for flexion/ extension radiography.

The association of vertebral instability with changes in the bone marrow adjacent to the endplates has been discussed, but without consistent results [22-24]. Degenerative disk disease and facet joint osteoarthritis affect the stability of the motion segment. However, the exact relationship between degenerative disk disease, facet joint osteoarthritis, and vertebral instability at MR imaging has not been defined [10].

Advancements in MR imaging technology, particularly the evolution of open MR imaging scanners, have provided new opportunities to investigate spinal kinematics, particularly vertebral instability [25-27]. However, the reported results are not really convincing, and despite continuous development of MR imaging equipment, essential problems still arise during attempts to perform examinations in upright posture for patients with spinal disorders [27, 28]. The overall examination time created severe pain problems. Motion artifacts and difficulties in reproducing the positioning between the sequences occurred regularly. This impaired the possibilities for analyzing the content of the spinal canal.

Radiostereometric analysis and distortion-compensated roentgen analysis

Radiostereometric analysis (RSA) has been proven to be the best method to evaluate three-dimensional translatory and rotatory motion between vertebrae [28]; unfortunately, this method is technically difficult, time-consuming, and requires specific apparatus. Moreover, because of its invasive nature, it is unsuitable for studies of large patient series. For this reason, there has been an interest in alternative noninvasive methods such as distortion-compensated roentgen analysis (DCRA) protocol. By using advanced methods of image analysis, DCRA measures rotation and translation in the sagittal plane from lateral flexion-extension radiographs of the lumbar spine. Leivseth et al. [29], measuring sagittal plane 
translatory and rotatory motion with DCRA and RSA in 15 lumbar segments of eight patients, found that measurement precision of DCRA is inferior to that of RSA but higher than that of conventional protocols assessing lumbar segmental motion.

\section{Clinical and radiologic correlations}

Diagnosing degenerative lumbar spine instability is difficult in the clinical setting. Overall, determination of the relationship between imaging instability and its symptoms remains challenging if not impossible. Pitkanen et al. [12] found that clinical signs of lumbar instability were poorly correlated with abnormalities found on functional radiographs. Dvorak et al. [30] found that the analysis of the segmental motion of the lumbar spine using functional radiographs does not aid in differentiating the underlying pathologic condition of a patient with LBP. Conversely, Iguchi et al. [31] found that the presence of translation $(\geq 3 \mathrm{~mm})$ and angulation $\left(\geq 10^{\circ}\right)$ could be an indicator for the persistence of the symptoms.

\section{Unsolved issues}

Primary vertebral instability as a cause of chronic LBP emerged as a seemingly distinct clinical entity almost 50 years ago. Since then, the diagnostic label "segmental instability" has been criticized, as it lacks scientific evidence of being diagnosable with accuracy in LBP sufferers. In patients who do have LBP, there are no agreed signs and symptoms that can be truly attributable to instability. This inability to properly diagnose instability despite better imaging techniques and methods of testing spinal instability has led to some authors requesting that the term "instability" be abolished, as it is an unproven label. In addition, this inability to accurately diagnose instability accounts for a variable response to the various conservative and surgical therapeutic options.

Surgery for pain in spinal instability has a wide spectrum of options, and the surgeon's preference is probably the most important factor governing the choice that is offered to the patient. Surgery relies on medicine's strong tradition of empiricism and survives because of its perceived beneficial effects, rather than as a result of any wellfounded rationale. Thus, although a scientific rationale for the treatment of instability is highly desirable, it is important to bear in mind that the ultimate goal of this kind of surgical intervention is to alleviate pain. First, fusion is the most commonly offered procedure that can be performed anteriorly, posteriorly, posterolaterally, or in combination. In addition to these different approaches are allied a huge variety of surgical internal fixation devices for stabilization. The objective here is to fix the unstable segment which will, if successful and solid, become painless [32]. The second alternative is to restore stability but retain mobility of the segment by flexible stabilization such as the Graf ligament using tension banding and pedicle screws [33]. Third, a number of disk replacement prostheses have been promoted to restore the necessary structural and biomechanical properties of the spine that need to exist to achieve lumbar lordosis and stability of the segmental motion segments [34].

In an age when both patient and doctor would like in principle to follow evidence-based practice, significant limitations, deficiencies, and difficulties still persist. There is no sensitivity, specificity, predictive value, or accuracy of the tests used in establishing instability in the papers that report the outcome following spinal surgery for instability [32].

Imaging has been harnessed to play an increasing role in the diagnosis and management of patients with suspected instability. The pathological changes in the spine in the three phases postulated by Kirkaldy-Willis and Farfan [4] in 1982 can be accurately identified, but how they relate to the functional concepts of dysfunction, instability, and stabilization is not always clear. These pathological changes along with the complications of disk herniation and spinal stenosis have also all been demonstrated in asymptomatic individuals. To complicate matters further, it is generally agreed that the physical disorder of segmental instability is associated with an emotional and/or psychological reaction which also needs to be recognized and treated appropriately to avoid an inferior therapeutic outcome [33]. The validated pain score forms and psychosocial abnormalities identified by the Oswestry Low Back Pain Disability Questionnaire [35], Short-Form 36 health survey questionnaire [36], and Distress and Risk Assessment Method [37] were far better than any radiographic, CT, MR imaging, ordinary clinical examination, or lumbar injection studies, including discography, for predicting the outcome of fusion operations [38].

All of this uncertainty and controversy creates an ethical burden for all doctors from all disciplines involved in the diagnosis and treatment of patients with LBP who are thought to be suffering from segmental instability. The history of spinal imaging is fraught with new findings felt to establish a clear anatomic cause of LBP illness: from simple "traction" spur to annular bulging and annular tears. So far, except for destructive lesions such as tumors, infections, and gross instability, the same findings are found much too frequently in subjects without LBP to be reliable independent diagnostic indicators.

Currently, imaging helps to select those patients who have supportive evidence of a cause-and-effect relationship in their spine that shows the degeneration process associated with segmental instability. It is, however, still far from 
satisfactory, with significant gaps in the knowledge that will formulate a unified concept of this condition. The quantification of normal and abnormal spinal motion is likely to be still dependent on imaging. It is unlikely that any future agreement of definition, clinical syndromes, and therapeutic regimes can be reached if clinically useful measurements are not a fundamental component of the whole concept of instability.

\section{References}

1. Pope MH, Panjabi M. Biomechanical definitions of spinal instability. Spine 1985; 10: 255-256.

2. Frymoyer JW, Selby DK. Segmental instability: rationale for treatment. Spine 1985; 10: 280-286.

3. Leone A, Guglielmi G, Cassar-Pullicino VN, Bonomo L. Lumbar intervertebral instability: a review. Radiology 2007; 245: 62-77 (review).

4. Kirkaldy-Willis WH, Farfan HF. Instability of the lumbar spine. Clin Orthop Relat Res 1982; 165: 110-123.

5. Dupuis PR, Yong-Hing K, Cassidy JD, Kirkaldy-Willis WH. Radiological diagnosis of degenerative lumbar spinal instability. Spine 1985; 10: 262-266.

6. Fujiwara A, Lim TH, An HS, et al. The effect of disc degeneration and facet joint osteoarthritis on the segmental flexibility of the lumbar spine. Spine 2000; 25: 3036-3044.

7. Grobler LJ, Robertson PA, Novotny JE, Pope MH. Etiology of spondylolisthesis: assessment of the role played by lumbar facet joints morphology. Spine 1993; 18: 80-92.

8. Vogt MT, Rubin D, Valentin RS, et al. Lumbar olisthesis and lower back symptoms in elderly white women: the study of osteoporotic fractures. Spine 1998; 23: 2640-2647.

9. Soini J, Antti-Poika I, Tallroth K, Konttinen YT, Honkanen V, Santavirta S. Disc degeneration and angular movement of the lumbar spine comparative study using plain and flexion-extension radiography and discography. J Spinal Disord 1991; 4: 183-187.

10. Murata M, Morio Y, Kuranobu K. Lumbar disc degeneration and segmental instability: a comparison of magnetic resonance imaging and plain radiographs of patients with low back pain. Arch Orthop Trauma Surg 1994; 113: 297-301.

11. Panjabi MM, White AA III. Physical properties and functional mechanics of the spine. In: White AA III, Panjabi MM, editors. Clinical biomechanics of the spine. Philadelphia, PA: Lippincott; 1978. p. $1-60$.

12. Pitkanen MT, Manninen HI, Lindgren KA, Sihvonen TA, Airaksinen O, Soimakallio S. Segmental lumbar spine instability at flexion-extension radiography can be predicted by conventional radiography. Clin Radiol 2002; 57: 632-639.

13. Pitkanen M, Manninen HI. Sidebending versus flexion-extension radiographs in lumbar spinal instability. Clin Radiol 1994; 49: 109-114.

14. Nizard RS, Wybler M, Laredo JD. Radiologic assessment of lumbar intervertebral instability and degenerative spondylolisthesis. Radiol Clin North Am 2001; 39: 55-71.

15. Wood KB, Popp CA, Transfeldt EE, Geissele AE. Radiographic evaluation of instability in spondylolisthesis. Spine 1994; 19: 16971703.

16. McGregor AH, McCarthy ID, Hughes SP. Motion characteristics of the lumbar spine in the normal population. Spine 1995; 20: 2421-2428.

17. Morgan FP, King T. Primary instability of lumbar vertebrae as a common cause of low back pain. J Bone Jt Surg Br 1957; 39-B: 6-22.

18. Shaffer WO, Spratt KF, Weinstein J, Lehmann TR, Goel V. The consistency and accuracy of roentgenograms for measuring sagittal translation in the lumbar vertebral motion segment. Spine 1990; 15: 741-750.

19. Posner I, White AA 3rd, Edwards WT, Hayes WC. A biomechanical analysis of the clinical stability of the lumbar and lumbosacral spine. Spine 1982; 7: 374-389.

20. Dvorak J, Panjabi MM, Chang D, Theiler R, Grob D. Functional radiographic diagnosis of the lumbar spine: flexion-extension and lateral bending. Spine 1991; 16: 562-571.

21. Trammell TR, Schroeder RD, Reed DB. Rotatory olisthesis in idiopathic scoliosis. Spine 1988; 13: 1378-1382.

22. Lang P, Chafetz N, Genant HK, Morris JM. Lumbar spinal fusion: assessment of functional stability with magnetic resonance imaging. Spine 1990; 15: 581-588.

23. Bram J, Zanetti M, Min K, Jodler J. MR abnormalities of the intervertebral disks and adjacent bone marrow as predictors of segmental instability of the lumbar spine. Acta Radiol 1998; 39: $18-23$.

24. Carragee EJ, Paragioudakis SJ, Khurana S. 2000 Volvo Award winner in clinical studies: lumbar high-intensity zone and discography in subjects without low back problems. Spine 2000; 25: 2987-2992.

25. Saifuddin A, Blease S, Macsweeney E. Axial loaded MRI of the lumbar spine. Clin Radiol 2003; 58: 661-671.

26. Weishaupt D, Schmid MR, Zanetti M, et al. Positional MR imaging of the lumbar spine: does it demonstrate nerve root compromise not visible at conventional MR imaging? Radiology 2000; 215: 247-253.

27. Wildermuth S, Zanetti M, Duewell S, et al. Lumbar spine: quantitative and qualitative assessment of positional (upright flexion and extension) MR imaging and myelography. Radiology 1998; 207: 391-398.

28. Johnsson R, Axelsson P, Stromqvist B. Posterolateral lumbar fusion using facet joint fixation with biodegradable rods: a pilot study. Eur Spine J 1997; 6: 144-148.

29. Leivseth G, Brinckman N, Frobin W, Johnsson R, Stromqvist B. Assessment of sagittal segmental motion in the lumbar spine: a comparison between distortion-compensated and stereophotogrammetric roentgen analysis. Spine 1998; 23: 2648-2655.

30. Dvorak J, Panjabi MM, Novotny JE, Chang DG, Grob D. Clinical validation of functional flexion-extension roentgenograms of the lumbar spine. Spine 1991; 16: 943-950.

31. Iguchi T, Kanemura A, Kasahara K, et al. Lumbar instability and clinical symptoms: which is the more critical factor for symptoms: sagittal translation or segment angulation. J Spinal Disord Tech 2004; 17: 284-290.

32. Hanley EN Jr. The indications for lumbar spinal fusion with and without instrumentation. Spine 1995; 20(24 suppl): 143S-153S.

33. Markwalder TM, Wenger M. Dynamic stabilization of lumbar motion segments by use of Graf's ligaments: results with an average follow-up of 7.4 years in 39 highly selected, consecutive patients. Acta Neurochir (Wien) 2003; 145: 209-214.

34. Sears W. Posterior lumbar interbody fusion for degenerative spondylolisthesis: restoration of sagittal balance using insert-androtate interbody spacers. Spine J 2005; 5: 170-179.

35. Fairbank JC, Couper J, Davies JB, O'Brien JP. The Oswestry low back pain disability questionnaire. Physiotherapy 1980; 66: 271273.

36. Garratt AM, Ruta DA, Abdalla MI, Buckingham JK, Russell IT. The SF36 health survey questionnaire: an outcome measure suitable for routine use within the NHS. BMJ 1993; 306: 1440-1444.

37. Main CJ, Wood PLR, Hollis S, Spanswick CC, Waddell G. The distress and risk assessment method: a simple patient classification to identify distress and evaluate the risk of poor outcome. Spine 1992; 17: 42-52.

38. Nachemson AL. Instability of the lumbar spine: pathology, treatment, and clinical evaluation. Neurosurg Clin N Am 1991; 2: 785-790. 